Pediat. Res. 4: 510-521 (1970)

DISGUSSION OF ABSTRAGTS OF

\title{
Meeting of The American Pediatric Society
}

\author{
Atlantic City, New Jersey, April 30, 1970
}

Plenary Sessions ${ }^{1}$

Role of Nasopharyngeal Tonsils in the Mechanism of Mucosal Immunity to Poliovirus. Pearay L. Ogra, Dept. of Ped., State Univ., of New York at Buffalo (introduced by David T. Karzon).

Richard W.Newcomb (Children's Asthma Research Institute, Denver, Colo.): Do you have any idea how the antibody titers in your nasal fluids relate to immunoglobulin concentrations?

Dr. Ogra: We are working on it at the moment. It seems that total gamma-A-immunoglobulin concentration decreases by about twofold, but it is not as constant as the drop in the specific viral antibody.

E. Richard STIEHM (UCLA Center for the Health Sciences) : What is the relative contribution of the tonsillectomy and the adenoidectomy to the immune deficiency noted?

Dr. OGRA: It is very hard these days to pick up children who have had isolated tonsillectomies. I do not have any information on the individual participation of tonsils and adenoids. Most of the children we studied had complete removal of tonsils as well as adenoids.

2 A Controlled Evaluation of the Effectiveness of Comprehensive Pediatric Care in Influencing Patient Compliance. LeOn Gordis and Milton Markowitz, Hebrew Univ.-Hadassah Med. Sch., Jerusalem, and Univ. of Conn. Sch. of Med. Hartford Conn.

Alvin H. Novack (New Haven, Conn.): Dr. GorDIs, $I$ want to indicate that it is important that we measure the differences between comprehensive and episodic care. I would, however, raise a question as to the definition of comprehensive care. I think you have measured the difference between comprehensive physician care versus episodic physician care. Comprehensive health care must be defined more broadly than just physician care and should include social and psychiatric (mental health) in addition to the usual medical, nursing, and dental health care.

Secondly, it is important to consider the difference between comprehensive and episodic care. The latter is disease oriented, and the former is health promoting, and it is difficult to compare the two when one talks about a chronic disease and compliance.

Dr. GoRDIs: In response to your first question, it seems to me that in analyzing comprehensive care we have to study each of the components that go into iteach component individually as well as all the components together - if we are to find which factors are critical and which factors are extraneous. Although it is

${ }_{1}$ Abstracts appeared in Pediat. Res. 4: 371-374 (1970). true that this study focused only on comprehensive care by a physician, the care was not restricted to a single organ system or set of problems. But I am in full agreement with you that different types of studies should also be done, including those of other health professionals, and in different settings.

As far as it being disease oriented, I agree with you there, too. The problem is that the research in this area cannot be done cosmically; we must isolate the specific factors which we wish to analyze, and submit them to rigorous evaluation. I would suggest, therefore, that this type of study should be extended to preventive health services and health care, as well as to chronic disease management.

WILlIAM OBRINSKy (Montefiore Hospital, NewYork, N.Y.): Comprehensive care is a way of life. It is not something that you can decide on one day to turn on, and a year later to turn off. You have had a population that has never had any experience with comprehensive care, and it takes a lot longer than 1 year to begin to teach the principles of comprehensive care. I would be very much interested in a similar study that might be done with a population who from very early infancy had comprehensive care over a longer period than 1 year.

Joel J.Alpert (Children's Hospital Medical Center, Harvard Medical School, Boston, Mass. ) : Dr. GorDrs, I agree with your expressed philosophy very much. We need controlled evaluations of comprehensive care. The challenge, however, is not only to try to measure differences but also to offer explanations as to why differences were or were not found. Thus, other points might be that these were families that were engaged in an identical physical place; that this was care given to individual patients and not to families.

Your report is very similar to some of our own work. We did not measure differences in the first year of our study on 551 families but saw differences in the second and third.

We also found that many of these measured differences disappeared in the third year of the study and these disappearing differences were due to those families on welfare who were pulled back into what we call the welfare-fragmented system by pressures outside of the comprehensive care program. What may, indeed, be needed is a total change in the health care system. Perhaps you have also identified a very complex population. I wonder how many of your 77 patients were on welfare.

Dr. GoRDIs: Only a relatively small proportion were on welfare, but the group was too small to be able to subject it to this type of analysis. 
I would like to make it clear that we are not suggesting that the lack of positive findings here applies to comprehensive care programs in general. We suggest only that this approach be used to submit comprehensive care to a truly rigorous evaluation of its effectiveness.

Barbara M. Korsch (Children's Hospital, Los Angeles, Calif.): If, as has been stated, this may not have been an example of truly comprehensive care, in that it was not delivered by a health team and did not start at birth, on the other hand, it did seem to isolate the factor of continuous medical care by one physician. Here again, it seems to me that there are other studies, like the one by CHARNEY et al. where continuous care by a particular pediatrician did seem to make for increased compliance with various medical regimes studied previously.

So it is not a simple situation, even if you say that you are simply introducing the factor of the continuous relationship with a doctor. The question is: Can you legislate this in a hospital setting at one moment, and produce a change, or can you not?

To document the study, you may need larger numbers of cases, because in our earlier work on compliance with medical advice we did have some very discourag ing months during the first few hundred cases, and finally the zeros before the ones began to appear after we were well beyond 600 or 700 patients. This was an outpatient department. I do not think that the findings in this number necessarily mean that you might not get a real difference.

There are several other points that interested me. would be very curious to do some studies on the actual interaction between the physicians in the two systems and their patients, because our work has shown some statistically significant differences in patients following medical advice which could be predicted on the basis of specific attributes of an individual interaction of a new physician with a new patient around an illness, and I would be curious to see how the physicians in the two groups relate to patients, and how they communicate with their patients.

Finally, lest someone get the impression that the fact of having a continuous relationship with a doctor like this does not improve patients' follow-through on medical advice. When we started our studies we had done some work that suggested that perhaps the social distance between the physician and the patient might make the patient more compliant, in the sense that he is a big authority, and you cannot get too close to him, and maybe what he says might be more important, and therefore you were anxious to do what he said. We found exactly the opposite to be true in our compliance study; namely, that effect, and friendliness, and conversation other than strictly medical conversation, had a positive effect on compliance.

EDGAR J.SCHOEN (Kaiser Foundation Hospital, Oakland, Calif.): The authors considered they were delivering comprehensive care. Did the patients?

You mentioned that two senior residents were used. What was the total number of physicians involved in this work? Also, at the end of the study, were the patients asked at any point if they knew the name of their doctor?

Dr. GoRdis: The same two physicians provided all the care for the comprehensive care group. In response to your second question, on the identification of the physician by the patient, there were significant differences between the two groups in the percentage of who could correctly identify their physician by name.
Nigholas M. Nelson (Boston, Mass.): I would like to suggest that your slide which showed an increase in noncompliance in both the experimental and control groups is yet another demonstration of the uncertainty principle, or as it is sometimes known, the Hawthorne effect; namely, that it is impossible to observe the phenomenon without changing the phenomenon observed.

President DAy: I think we will have to close the discussion.

3 Differences in Glycolysis Between Fetal and Non-fetal Fibroblast Cell Lines. AntoinetTe Condon, Frank A. Oski, Salvatore Di Mauro and William J. Mellman, Depts. of Ped., Neurol. and Med. Genet., Univ. of Pennsylvania.

Wallace W. MaGrory (New York Hospital, Cornell Medical Center, NewYork, N.Y.) : The demonstration of a difference in channeling of glucose metabolism into the hexose-monophosphate shunt and the KrebsTCA pathway in fetal and adult cells in tissue culture is in agreement with similar findings of Dr. E. ScHUBERT in the Department at Cornell. Dr. Sarubert's studies were done on kidney tissue in differing periods from fetal life to maturity in the rat. The changes correlate with the growth program of the organ with maturation. The activity of the hexose-monophosphate shunt is highest in proliferating cells and falls to adult values when growth by hyperplasia ceases (about 40 days after birth). What I find especially interesting is your data showing fetal cells continue to demonstrate this metabolic pattern in tissue culture if $\mathrm{I}$ understood your comments. Did you find that fetal cells remained 'fetal' in this regard in serial tissue culture?

Miss Condon: Yes.

Leonard Pinsky (Lady Davis Institute, Montreal, Que.): Miss ConDon and her associates have shown us once again that cultured human fibroblasts are not the dedifferentiated masses they were thought to be a few years ago. In other words, the age of the donor and, perhaps, the anatomic site of the parental tissue may confer heritable properties on the cell strain developed.

I wonder if the speaker has had any experience with newborn fibroblasts. I have the feeling that fibroblasts derived from newborns, and particularly newborn foreskin, have many of the properties she has described for her fetal strains. It would be important to know if this is true because one frequently sees cell strains derived from human foreskin used as controls in studies dealing with fibroblasts cultured from adult skin.

Miss Condon: We have no experience with foreskin from newborns.

4

Prenatal Diagnosis of the Mucopolysaccharidoses by a Chemical Method. Reuben Matalon, C. B. JACOBSON and Albert Dorfman. Univ. of Chicago, Dept. of Ped. and the Joseph P. Kennedy, Jr. Mental Retardation Res. Center, Chicago; and George Washington Univ. Med. Center, Washinton, DC.

IRWIN A. SaHAFER (Cleveland Metropolitan General Hospital, Cleveland, Ohio): Dr. TAKEUGH in our laboratory has been working with amniotic fluid. She has analyzed a much smaller number of normal controls, but can confirm your results with one minor exception. We find in normal amniotic fluid a small amount of heparitin sulfate. I think chemically the material that we get off the column is not as yet as well characterized as some of the material with which you have worked. 